\title{
The State of Strain in Single GaN Nanocolumns As Derived from Micro-Photoluminescence Measurements
}

\author{
Nicolas Thillosen, ${ }^{\dagger}$ Kathrin Sebald, ${ }^{\ddagger}$ Hilde Hardtdegen, ${ }^{*}, \dagger$ Ralph Meijers, ${ }^{\dagger}$ \\ Raffaella Calarco, ${ }^{\dagger}$ Simone Montanari, ${ }^{\dagger}$ Nicoleta Kaluza, ${ }^{\dagger}$ Jürgen Gutowski, ${ }^{\ddagger}$ and \\ Hans Lüth ${ }^{\dagger}$
}

Institute of Thin Films and Interfaces (ISG1), CNI (Center of Nanoelectronic Systems

for Information Technology), Research Center Jülich, 52425 Jülich, Germany, and

Institute of Solid State Physics, University of Bremen, P.O. Box 330440,

D-28334 Bremen, Germany

Received December 13, 2005; Revised Manuscript Received February 17, 2006

\begin{abstract}
In the present paper, studies on the state of strain in single and ensembles of nanocolumns investigated by photoluminescence spectroscopy will be presented. The GaN nanocolumns were either grown in a bottom-up approach or prepared in a top-down approach by etching compact GaN layers grown on $\mathrm{Si}(111)$ and sapphire (0001) substrates. Experimental evidence for strain relaxation of the nanocolumns was found. The difference and development of the strain value for different nanocolumns could be verified by spatially resolved micro-photoluminescence on single nanocolumns separated from their substrate. A common $\mathrm{D}^{0} \mathrm{X}$ spectral position at $3.473 \mathrm{eV}$ was found for all separated single GaN nanocolumns independent of the substrate or processing technique used, as expected for a relaxed system.
\end{abstract}

Introduction and Experimental Setup. Besides carbon nanotubes, semiconductor nanocolumns or whiskers are possible candidates for upcoming nanoelectronics which will complement or, in niches, replace Si ultrahigh integration CMOS technology when this technique will reach its physical limits within the next 15 years. Semiconductor nanocolumns with diameters of several tens of nanometers can be grown epitaxially in a self-organized bottom-up way without the use of cost consuming nanolithography. So far, there are several reports on the fabrication of $\mathrm{GaN}$ nanowires and nanorods ${ }^{1-9}$ and on the optically pumped lasing of such structures. ${ }^{8,24}$ Furthermore, in contrast to the case of carbon nanotubes, the whole semiconductor heterostructure technology can easily be transferred to the growth of nanocolumns such that complex quasi-one-dimensional heterostructure device architectures with incorporated barriers, quantum wells, and dots can be realized. Thus, quantum electronic networks and, eventually, the realization of quantum computers come one step further toward realization. An essential ingredient to these ideas is the possibility to realize heterostructure nanocolumns with barriers and wells of material combinations which are not well-lattice-matched and there-

* To whom correspondence may be addressed. E-mail: h.hardtdegen@ fz-juelich.de.

$\dagger$ Institute of Thin Films and Interfaces (ISG1), CNI (Center of Nanoelectronic Systems for Information Technology), Research Center Jülich.

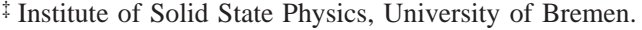

fore do not grow with crystallographic perfection in extended layer structures. Indeed, due to the lack of commercially available free-standing $\mathrm{GaN}$ substrates, heteroepitaxial growth has to be carried out on alternative substrates which provide, beside the inherent lattice mismatch, additionally, different thermal expansion coefficients, the latter inducing stress in the $\mathrm{GaN}$ during the postepitaxial cooling down of the sample. Even if several hundreds of micrometers of $\mathrm{GaN}$ are deposited on sapphire, so that strain is expected to approach zero with increasing thickness, a strong strain-inducing bowing is observed. ${ }^{14}$ While during layer growth the poorly lattice matched systems are not able to relax into perfect crystals, the nanocolumn's dimension should allow for local relaxation even for not well adapted material combinations such as GaN/AlGaN, GaAs/InAs, etc. This crystallographic relaxation of semiconductor nanocolumns has been expected, and some experimental results support this idea. ${ }^{10-13}$ The observation of a strain convergence by realizing nanocolumns on different lattice-mismatched substrates would open the possibility to determine physical data for fully relaxed GaN. In the present paper experimental evidence for strain relaxation effects is demonstrated for $\mathrm{GaN}$ nanocolumns by the analysis of the donor-bound exciton transition $\left(\mathrm{D}^{0} \mathrm{X}\right)$ energy in photoluminescence (PL) spectra of single column samples grown on different substrates. The $\left(\mathrm{D}^{0} \mathrm{X}\right)$ luminescence peak energy being a measure for the band gap energy (obtained by adding the bound-exciton total binding energy) 


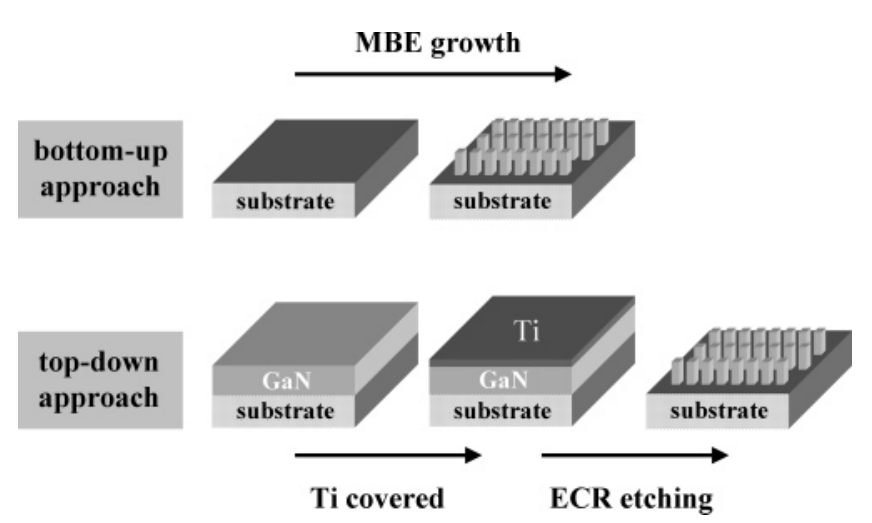

Figure 1. Bottom-up (a) and top-down (b) approach for the preparation of nanocolumns.

is strongly sensitive to the state of strain within the sample. ${ }^{15}$ Thus we compare PL spectra of GaN nanocolumns which have been grown on a $\mathrm{Si}(111)$ substrate under tensile strain with those which were prepared by etching from GaN layers grown on $\mathrm{Si}(111)$ and sapphire substrates under tensile and compressive strain, respectively.

$\mathrm{GaN}$ growth on $\mathrm{Si}(111)$ substrates was carried out by plasma-assisted molecular beam epitaxy using $\mathrm{N}_{2}$ as nitrogen source. For compact bulk GaN samples, growth was performed under Ga-rich conditions at a substrate temperature of $770{ }^{\circ} \mathrm{C}$. The GaN nanocolumns which were prepared in a bottom-up approach-as shown in Figure 1-needed N-rich conditions and a growth temperature of $800{ }^{\circ} \mathrm{C} .{ }^{16,17} \mathrm{GaN}$ growth on sapphire substrates was carried out by metalorganic vapor phase epitaxy at $1100{ }^{\circ} \mathrm{C}$ with trimethylgallium and $\mathrm{NH}_{3}$ as precursors. Only GaN bulk growth was performed by this method. The top-down type of nanocolumns was prepared by first cleaning the respective bulk GaN layer surfaces with an $\mathrm{O}_{2}$ plasma and standard wet etching before evaporating $300 \mathrm{~nm}$ of Ti. Finally, the titanium metal mask was etched by using the electron-cyclotron-resonance reactive-ion-etching (ECR-RIE) method for $17 \mathrm{~min}$ with a $\mathrm{Cl}_{2}$ and Ar gas mixture at $280 \mathrm{~W}$ microwave power and with $55 \mathrm{~W}$ radio frequency power. Under these conditions nonuniform etching of the Ti layer is observed. Where the titanium was removed also, the underlying $\mathrm{GaN}$ was etched leading to nanocolumns underneath the metal, as presented schematically in Figure 1. Finally, by further etching, the metal on top of the nanocolumns was completely removed. Scanning electron microscopy (SEM) images taken from the nanocolumn samples are shown in Figure 2. The etched nanocolumns on Si substrates are inhomogeneously distributed over the wafer. Furthermore they get thinner with increasing height (base-to-tip ratio 10), reaching diameters in the order of $50 \mathrm{~nm}$ for the thinnest ones (Figure 2a). The bottom-up grown nanocolumns on Si substrates are isolated and possess a hexagonal shape with typical diameters, heights and densities of 50-80 nm, $0.1-0.5 \mu \mathrm{m}$, and $(3-4) \times 10^{9}$ $\mathrm{cm}^{2}$, respectively (Figure $2 \mathrm{~b}$ ). For nanocolumns prepared by etching GaN/sapphire samples, the column density is about $(1-2) \times 10^{9} \mathrm{~cm}^{2}$ with a homogeneous length of approximately $1 \mu \mathrm{m}$ and a width in the order of 50-70 nm. The base-to-tip ratio is in the order of 2-3 (Figure 2c); thus,

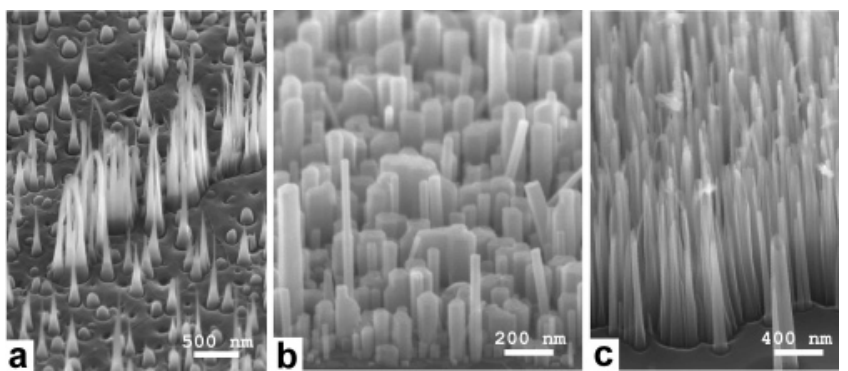

Figure 2. SEM of GaN/Si(111) nanocolumns etched (a), as grown (b), and of GaN/sapphire etched (c).

the conicity is much less than that for the previously described GaN/Si(111) etched nanocolumns.

From the SEM images it becomes obvious that for the investigated nanocolumns quantum-confinement effects do not play any role for the optical characteristics of the nanocolumns since their typical diameter of $\geq 50 \mathrm{~nm}$ is at least five times the exciton Bohr radius in $\mathrm{GaN}$.

The GaN bulk samples on $\mathrm{Si}(111)$ and sapphire were investigated using a standard (non-spatially-resolved) PL setup. For ensembles of nanocolumns and single nanocolumns, the samples were studied by micro-photoluminescence $(\mu$-PL) measurements. They were mounted in a $\mathrm{He}$ flow cryostat allowing temperatures between 4 and $300 \mathrm{~K}$. Measurements presented here were carried out at $T=4 \mathrm{~K}$. The samples were excited with the $325 \mathrm{~nm}$ line of a $\mathrm{HeCd}$ laser. The laser beam was focused by a microscope objective with a numerical aperture of 0.5 to a spot size of about 1.5 $\mu \mathrm{m}$ on the sample. The PL was collected by the same objective and dispersed by a $1 \mathrm{~m}$ monochromator. The $\mu$-PL signal was detected by a liquid-nitrogen-cooled CCD camera. The spectral resolution of the experimental setup was in the order of $0.17 \mathrm{meV}$. To measure $\mu$-PL on single nanocolumns, a few nanocolumns were separated mechanically from their original substrate and deposited on another substrate. Since the energy separation between the heavy, light, and crystalfield split-off valence band is related to the strain in $\mathrm{GaN},{ }^{15}$ the photoluminescence excitonic line positions such as $E_{\mathrm{D}^{0} \mathrm{X}}$ give direct insight into the lattice-strain state of the GaN sample. This is especially useful as it allows relaxation processes observed in nanocolumns to be monitored.

Experimental Results and Discussion. (A) Etched Nanocolumns on $\mathbf{S i ( 1 1 1 ) . ~ I n ~ F i g u r e ~} 3$ the comparison between the PL spectrum of the GaN/Si(111) bulk (dotted line) and the $\mu$-PL measurements of the corresponding ensemble of etched nanocolumns (blue line) and a single nanocolumn (black line) is displayed on a logarithmic scale. For the single nanocolumn a representative emission spectrum is shown; for some nanocolumns defect-related emission peaks can be observed as well. Because of the different excitation conditions of the compact layer and the nanocolumn ensemble, two different scales for the measured intensity signals had to be used. Nevertheless, it is possible to compare the relative signal intensities of the bulk material to the nanocolumn ensemble because standard PL measurements have been performed as a reference on both samples under the same excitation conditions. A considerable increase of 


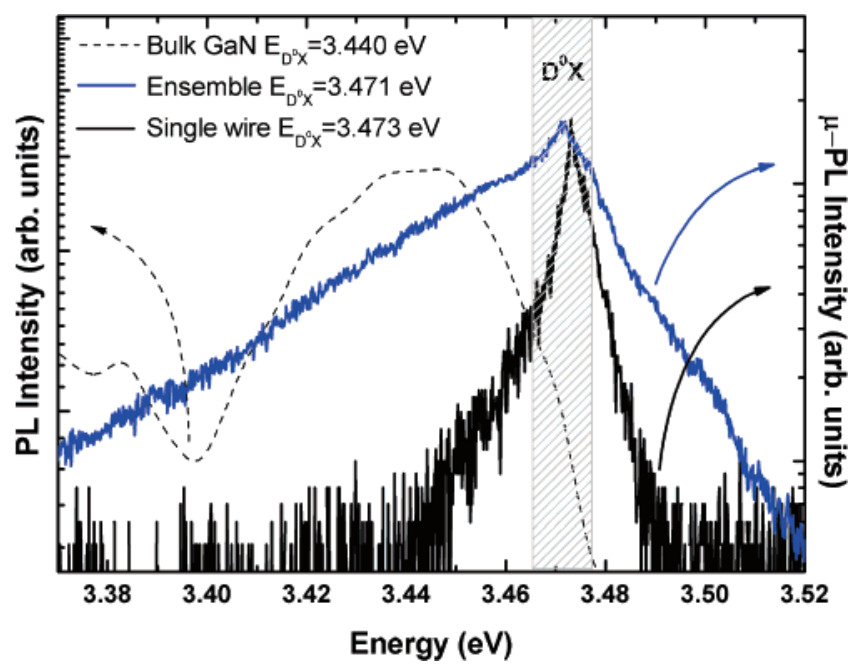

Figure 3. Bulk GaN/Si(111) (dotted). Spatially resolved $\mu$-PL at $4 \mathrm{~K}$ of nanocolumns etched from the $\mathrm{GaN}$ layer on $\mathrm{Si}(111)$, ensemble (blue) and single column (black) structures (intensities are normalized). The dashed region from 3.466 to $3.476 \mathrm{eV}$ indicates literature values presumed for fully relaxed $\mathrm{GaN}$.

the intensity of the $E_{\mathrm{D}^{0} \mathrm{X}}$ excitonic emission peak by a factor of $10^{3}$ with respect to the unetched bulk sample was observed just after etching the bulk sample into nanocolumns. However, one has to keep in mind that two physical origins for the difference exist, (1) a larger surface in the nanocolumn case and (2) a different crystalline quality of the layers and the columns. We now focus on the PL transition energies of the donor-bound exciton $E_{\mathrm{D}^{0} \mathrm{x}}$ observed for the different samples as they are related to the corresponding strain states. The bulk GaN on $\operatorname{Si}(111)$ shows an emission energy $E_{\mathrm{D}^{0} \mathrm{X}}$ of $3.440 \mathrm{eV}$. For the nanocolumns etched from the bulk GaN on $\mathrm{Si}(111)$, a quite different emission energy of $E_{\mathrm{D}^{0} \mathrm{x}}=3.471$ $\mathrm{eV}$ is found. Concerning the nanocolumn detached from the substrate material, a further shift to $E_{\mathrm{D}^{0} \mathrm{x}}=3.473 \mathrm{eV}$ was measured. For totally relaxed GaN, there is still a large uncertainty concerning the corresponding emission energies of the $E_{\mathrm{D}^{0} \mathrm{X}}$ so that supposed literature values are found between 3.466 and $3.476 \mathrm{eV} .{ }^{18-22}$ This large spectral range for presumed fully relaxed $\mathrm{GaN}$ is represented as a dashed region in the following graphs. $\mathrm{D}^{0} \mathrm{X}$ energy values on the lower energy side of the dashed region are characteristic for GaN being under tensile strain and, therefore, having a smaller band gap compared to relaxed GaN. ${ }^{15}$ In the case of bulk $\mathrm{GaN} / \mathrm{Si}(111)$ the value $E_{\mathrm{D}^{0} \mathrm{X}}=3.440 \mathrm{eV}$ indicates that tensile strain is induced by the $\mathrm{Si}(111)$ substrate. The observed increase of the $E_{\mathrm{D}^{0} \mathrm{X}}$ emission energy for the nanocolumn ensemble sample and the single nanocolumn indicates stress relaxation. The separation of the nanocolumns from their strain-inducing substrate could explain the additional $E_{\mathrm{D}^{0} \mathrm{X}}$ emission energy shift of $2 \mathrm{meV}$. The value of $E_{\mathrm{D}^{0} \mathrm{x}}=3.473$ $\mathrm{eV}$ for a single and detached nanocolumn is expected to nearly correspond to fully relaxed GaN. Note that the measurements on the single nanocolumns were generally performed by exciting at half their height. $\mu$-PL measurements carried out at the base of the single nanocolumn showed in some cases a remaining strain indicated by a smaller $E_{\mathrm{D}}{ }^{0} \mathrm{X}$ emission energy. However, due to the relatively large

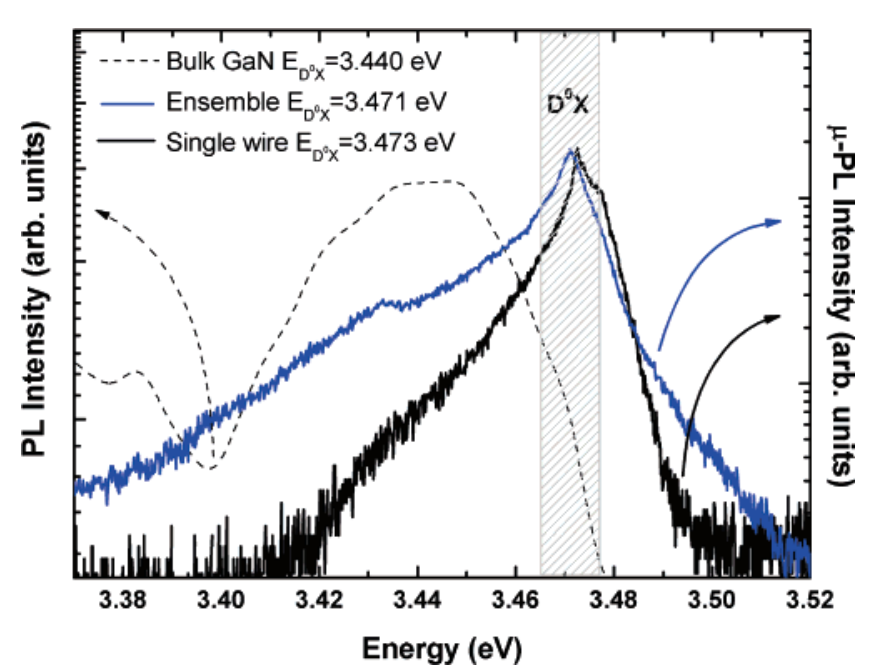

Figure 4. Bulk GaN/Si(111) (dotted). Spatially resolved $\mu$-PL at $4 \mathrm{~K}$ of nanocolumns grown on $\mathrm{Si}(111)$, ensemble (blue) and single column (black) structures (intensities are normalized). The shoulder at $3.477 \mathrm{eV}$ is assigned to the free $\mathrm{A}$ exciton. The dashed region from 3.466 to $3.476 \mathrm{eV}$ indicates literature values presumed for fully relaxed $\mathrm{GaN}$.

excitation laser spot size of $1.5 \mu \mathrm{m}$ the spatial distribution of the strain within a single nanocolumn cannot be analyzed.

The poor strain homogeneity of the unstructured $\mathrm{GaN}$ grown on $\mathrm{Si}(111)$ is confirmed by the large full width at half-maximum of the $\mathrm{D}^{0} \mathrm{X}$ being in the order of $50 \mathrm{meV}$. The etched nanocolumn ensemble exhibits an already reduced line width of $14 \mathrm{meV}$ measured by $\mu$-PL. The line width of the single nanocolumn $\mathrm{D}^{0} \mathrm{X}$ (black line) shows an even more reduced value of $4 \mathrm{meV}$. This observation is indeed consistent with the fact that measuring PL from a limited excited GaN volume (this is the case for $\mu$-PL measurements on nanocolumns) reduces the spectral broadening caused by structural inhomogeneities of the sample.

(B) Grown Nanocolumns on $\mathrm{Si}(111)$. The experimental findings for the nanocolumns grown in a self-assembled mode on Si are comparable to the observations on the etched nanocolumns. From the reference standard PL measurement, an even larger increase of the PL intensity of the $E_{\mathrm{D}^{0} \mathrm{X}}$ emission of the grown nanocolumns is observed when compared to the bulk layer, being on the order of $10^{4}$. In Figure 4 , the $E_{\mathrm{D}^{0} \mathrm{X}}$ emission peak of the ensemble spectrum is likewise shifted to higher energies when compared to the bulk layer on $\mathrm{Si}$ (compare section A), and for the single nanocolumn the emission energy of $3.473 \mathrm{eV}$ fully corresponds to that of the previously reported single column detached from the top-down etched sample. Further, a shoulder at $3.477 \mathrm{eV}$ can be recognized and assigned to the A-exciton transition. The contribution of both transition peaks results in an emission band being broader than the PL peak of the single nanocolumn shown in Figures 3 and 5 . Nevertheless, the observation of the free A exciton for the grown single nanocolumns indicates a higher crystal quality with fewer defects. In contrast to the case of the etched nanocolumn ensemble the line width of the as-grown ensemble has already decreased to $8 \mathrm{meV}$ which can be attributed to a better homogeneity within the ensemble of 


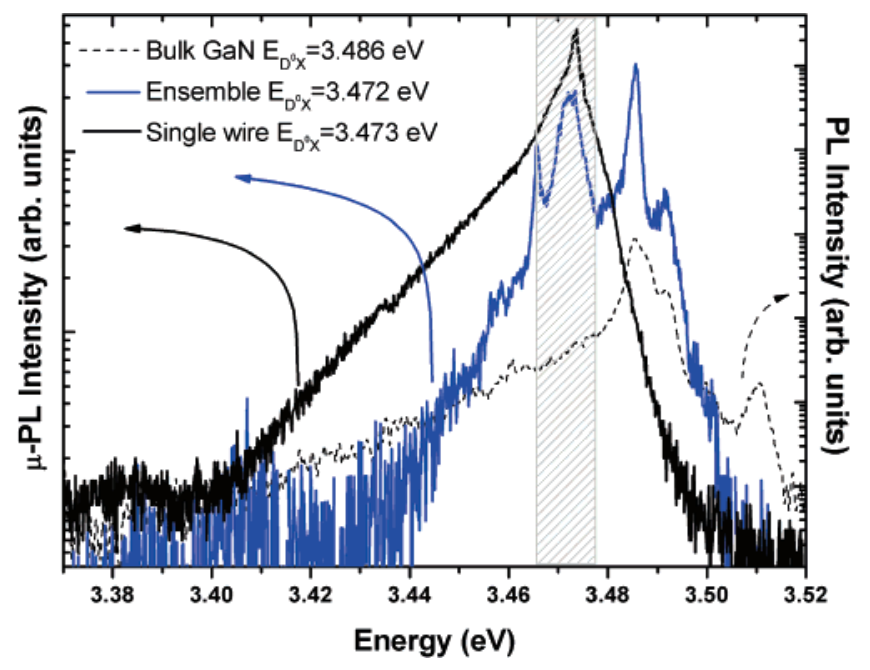

Figure 5. Bulk GaN/sapphire (dotted). Spatially resolved $\mu$-PL at $4 \mathrm{~K}$ of nanocolumns etched from the GaN layer on sapphire: ensemble (blue) and single column (black) structures (intensities are normalized). The dashed region from 3.466 to $3.476 \mathrm{eV}$ indicates literature values presumed for fully relaxed GaN.

bottom-up grown nanocolumns. Indeed, this value is in the same order as the one found previously for the single etcheddown nanocolumn $(4 \mathrm{meV})$. This fits the expectation that a bottom-up epitaxial growth technique for nanocolumns should induce much less inhomogeneity than column formation making use of etching techniques.

(C) Etched Nanocolumns on Sapphire. In Figure 5 the standard PL spectrum of bulk GaN on sapphire, the $\mu$-PL spectrum of the ensemble and that of a single nanocolumn on sapphire are displayed. From the reference standard PL measurement on the bulk material and the nanocolumn ensemble, a relative decrease of the $\mathrm{D}^{0} \mathrm{X}$ nanocolumn PL intensity was observed with respect to the bulk sample. This finding is in contrast to the results of Wang et al., ${ }^{11}$ which is most probably due to the different etching conditions (physical versus chemical effect with more or less plasmainduced surface damage). Sapphire substrates commonly induce biaxially compressive strain in bulk GaN samples. The consequence is an increase of the band gap and, therefore, an increase of the donor-bound exciton transition energy..$^{5}$ This fact is confirmed in Figure 5 showing a transition energy for bulk $\mathrm{GaN}$ on sapphire of $E_{\mathrm{D}^{0} \mathrm{X}}=3.485$ $\mathrm{eV}$, on the high-energy side of the dashed region representing fully relaxed $\mathrm{GaN}$. The line width of the $\left(\mathrm{D}^{0} \mathrm{X}\right)$ peak measured with standard PL amounts to $4 \mathrm{meV}$, which is much less than those obtained for the previously measured layers on $\mathrm{Si}(111)$ substrates. This is an indication of the good homogeneity and crystalline quality of the sample. The ensemble of etched-down nanocolumns (blue line) exhibits four different emission peaks. The two transitions at the higher energies of 3.485 and $3.491 \mathrm{eV}$ match with the two transitions originating from the bulk GaN (dotted line). As in this case the samples were not etched down to the sapphire substrate; a $2.5 \mu \mathrm{m}$ thick GaN buffer layer still remains. This explains why the PL originates not only from the nanocolumns but also from the underlying unetched and compressively strained GaN buffer layer. These PL transitions are therefore of the same origin as those measured before etching. They can be assigned to the same donor-bound exciton $(3.485 \mathrm{eV})$ and A exciton $(3.491 \mathrm{eV})$, respectively. The emission centered at $3.472 \mathrm{eV}$ with a larger spectral width may be due to the superposition of different transitions, as it shows at least two maxima. The transition at $3.473 \mathrm{eV}$ can be assigned to the $\mathrm{D}^{0} \mathrm{X}$, as it is matching with the $E_{\mathrm{D}^{0} \mathrm{X}}$ line being dominant for the single detached nanocolumn (black line with a peak line width of $2.6 \mathrm{meV}$ ). The doublet at lower energies (3.4715 and $3.4721 \mathrm{eV}$ ) possibly corresponds to excitons at $\mathrm{O}_{\mathrm{N}}$ and $\mathrm{Si}_{\mathrm{Ga}}$, respectively. ${ }^{23}$ The fourth main transition at $3.465 \mathrm{eV}$ is no longer present in the spectra of the single detached nanocolumn, so that it may be attributed to an ensemble-related defect transition. Finally, in a similar manner as described in sections $\mathrm{A}$ and $\mathrm{B}$, the shift of the $E_{\mathrm{D}^{0} \mathrm{X}}$ (but in this case toward lower energies) when starting from the compressively strained GaN buffer $(3.485 \mathrm{eV})$, and then looking for the ensemble and the single detached nanocolumn $(3.473 \mathrm{eV})$, is an indication for the relaxation of the compressive strain.

The $\left(\mathrm{D}^{0} \mathrm{X}\right)$ energy of the detached single nanocolumn is in any case found at $3.473 \mathrm{eV}$. Evidently and independent of the substrate or processing technique, the $\left(\mathrm{D}^{0} \mathrm{X}\right)$ energy for single detached nanocolumns fully corresponds to the value expected for a relaxed system.

Summary and Conclusions. GaN nanocolumns were prepared on sapphire and on $\mathrm{Si}(111)$ substrates either in a top-down approach by etching bulk $\mathrm{GaN}$ or in a bottom-up approach by growth. For all types of nanocolumn samples, single nanocolumns detached from the whisker lawn show a strong luminescence peak $\left(\mathrm{D}^{0} \mathrm{X}\right)$ at one and the same photon energy of $3.473 \mathrm{eV}$ independent of the preparation technique or the substrate used. This spectral position coincides with literature values which are expected for relaxed GaN. These findings demonstrate almost perfect strain relaxation of $\mathrm{GaN}$ nanocolumns if they are separated from their strain-inducing substrate. All in all it has been demonstrated that nanostructured material can be used to obtain physical data of fully relaxed $\mathrm{GaN}$, which is otherwise difficult to have access to.

Acknowledgment. The authors thank G. Crecelius for assistance with the low-temperature PL setup as well as H.P. Bochem and T. Richter for the SEM pictures. The Bremen group acknowledges support by the Deutsche Forschungsgemeinschaft in the framework of the Research Group 506, "Physics of Nitride-Based Nanostructured Light-Emitting Devices".

\section{References}

(1) Morales, A. M.; Lieber, C. M. Science 1998, 279, 208.

(2) Huang, Y.; Duan, X.; Chui, Y.; Lieber, C. M. Nano Lett. 2002, 2 , 101.

(3) Chen, C. C.; Yeh, C. C.; Chen, C. H.; Yu, M. Y.; Liu, H. L.; Wu, J. J.; Chen, K. H.; Chen, L. C.; Peng, J. Y.; Chen, Y. F. J. Am. Chem. Soc. 2001, 123, 2791.

(4) Tu, L. W.; Hsiao, C. L.; Chi, T. W.; LO, I.; Hsieh, K. Y. Appl. Phys. Lett. 2003, 82, 1601.

(5) He, M.; Zhou, P.; Mohammad, S. N.; Harris, G. L.; Halpern, J. B.; Jacobs, R.; Sarney, W. L.; Salamanca-Riba, L. J. Cryst. Growth 2001, $231,357$.

(6) Han, W.; Fan, S.; Li, Q.; Hu, Y. Science 1997, 277, 1287. 
(7) Birkhahn, R.; Hudgins, R.; Lee, D.; Steckl, A. J.; Molnar, R. J.; Saleh, A.; Zavada, J. M. J. Vac. Sci. Technol., B 1999, 17, 1195.

(8) Huang, M. H.; Mao, S.; Feick, H.; Yan, H.; Wu, Y.; Kind, H.; Weber, E.; Russo, R.; Yang, P. Science 2001, 292, 1897.

(9) Dhara, S.; Datta, A.; Wu, C. T.; Lan, Z. H.; Chen, K. H.; Wang, Y. L. Appl. Phys. Lett. 2004, 84, 3486.

(10) Macht, L.; Weyher, J. L.; Grzegorczyk, A.; Larsen, P. K. Phys. Rev. B 2005, 71, 073309.

(11) Wang, Y. D.; Chua, S. J.; Tripathy, S.; Sander, M. S.; Chen, P.; Fonstad, C. G. Appl. Phys. Lett. 2005, 86, 071917.

(12) Demangeot, F.; Gleize, J.; Frandon, J.; Renucci, M. A.; Kuball, M.; Peyrade, D.; Manin-Ferlazzo, L.; Chen, Y.; Grandjean, N. J. Appl. Phys. 2002, 91, 6520.

(13) Demangeot, F.; Gleize, J.; Frandon, J.; Renucci, M. A.; Kuball, M.; Peyrade, D.; Manin-Ferlazzo, L.; Chen, Y.; Grandjean, N. J. Appl. Phys. 2002, 91, 2866.

(14) Darakchieva, V.; Paskova, T.; Paskov, P. P.; Monemar, B.; Ashkenov, N.; Schubert, M. Phys. Status Solidi A 2003, 195, 516.

(15) Chuang, S. L.; Chang, C. S. Phys. Rev B 1996, 54, 2491.

(16) Calarco, R.; Marso, M.; Meijers, R.; Richter, T.; Aykanat, A. I.; Stoica, T.; Lüth, H. Proceedings of the ASDAM '04 Conference, Smolenice, Slovakia, 2004.
(17) Calarco, R.; Marso, M.; Richter, T.; Aykanat, A. I.; Meijers, R.; Hart, A. v.; Stoica, T.; Lüth, H. Nano Lett. 2005, 5, 981.

(18) Monemar, B. Phys. Rev. B 1974, 10, 676

(19) Kornitzer, K.; Ebner, T.; Thonke, K.; Sauer, R.; Kirchner, C.; Kamp, M.; Schwegler, V.; Leszczynski, M.; Grzegory, I.; Porowski, S. Phys. Rev. B 1999, 60, 1471.

(20) Volm, D.; Oettinger, K.; Streibl, T.; Kovalev, D.; Ben-Chorin, M.; Diener, J.; Meyer, B.; Majewski, J.; Eckey, L.; Hoffmann, A. Phys. Rev. B 1996, 53, 16543.

(21) Mayer, M.; Pelzmann, A.; Kamp, M.; Ebeling, K.; Teisseyre, H.; Nowak, G.; Leszczynski, M.; Grzegory, I.; Porowski, S.; Karczewski, G. Jpn. J. Appl. Phys., Part 2 1997, 36, L1634.

(22) Dingle, R.; Sell, D.; Stokowski, S.; Ilegems, M. Phys. Rev. B 1971, 4, 1211.

(23) Gogova, D.; Kasic, A.; Larsson, H.; Hemmingsson, C.; Monemar, B.; Tuomisto, F.; Saarinen, K.; Dobos, L.; Pecz, B.; Gibart, P.; Beaumont, B. J. Appl. Phys. 2004, 96, 799.

(24) Gradecak, S.; Qian, F.; Li, Y.; Park, H. G.; Lieber, C. M. Appl. Phys. Lett. 2005, 87, 173111.

NL052456Q 